Research Article

\title{
Research on Biomechanical Simulation and Simulation of Badminton Splitting and Hanging Action Based on Edge Computing
}

\author{
Bo Zhang (iD \\ College of Sports and Health, Guangzhou Sport University, Guangzhou 510500, China \\ Correspondence should be addressed to Bo Zhang; 51073@gzsport.edu.cn
}

Received 9 February 2021; Revised 15 April 2021; Accepted 17 April 2021; Published 27 April 2021

Academic Editor: Hsu-Yang Kung

Copyright (c) 2021 Bo Zhang. This is an open access article distributed under the Creative Commons Attribution License, which permits unrestricted use, distribution, and reproduction in any medium, provided the original work is properly cited.

\begin{abstract}
Sports biomechanics refers to the science of the laws of mechanical motion produced in the process of biological movement. Its essence is to systematically and digitally reconstruct the fundamental attributes and characteristics of motion. At present, the research of sports biomechanics mainly focuses on the theoretical research of basic aspects and lacks the new technology of sports biomechanics digital simulation innovation and data measurement. This article takes the badminton chopping action as the research object and carries out biomechanical simulation and simulation research with the help of edge computing and genetic algorithm. First of all, this paper constructs a badminton chopping and hanging action system framework based on edge computing, so as to facilitate simulation and improve data transmission efficiency. Secondly, genetic algorithm is used in biomechanics simulation and simulation optimization and data analysis process. System testing and simulation verify the excellent performance of the biomechanical simulation of badminton chopping and hanging action established in this paper. The research will provide a reference for the academic circles to explore the field of sports biomechanics.
\end{abstract}

\section{Introduction}

Sports biomechanics refers to the science of the laws of mechanical motion produced in the process of biological motion [1]. Its essence is to systematically and digitally reconstruct the fundamental attributes and fundamental characteristics of sports. Broadly speaking, sports biomechanics is the characteristics and evaluation of the state and attributes of all sports, and it is a comprehensive evaluation and analysis of all aspects of sports $[2,3]$. In a narrow sense, sports biomechanics is a study on the state of human motion. The object and main purpose of the study is human, and the mechanics in the basic category of physics is introduced into the concept of research. In short, sports biomechanics in the narrow sense is actually to simplify and objectify the connection activities of nerves, muscles, and joints in the process of human motion, as the gear link in the overall driving system, and only discuss the effects of this system $[4,5]$. The resulting force changes are analyzed and simulated in the digital form.
It is worth noting that the current sports biomechanics not only is a pure subject attribute and subject characteristic but also covers human anatomy, human biology, mechanics within the physical category, and psychology and other related fields. It is a comprehensive and academic direction with a multidisciplinary nature [6-8]. That is, from the perspective of fundamental properties, the essence of sports biomechanics is the combination of mathematics and mechanics, and its main research is to capture, reconstruct, analyze, and then judge the action based on the construction of mathematical models, computer, or model simulation [9]. Through the analysis of the force and angle in the athlete's sports state, the overall sports performance can be evaluated and improved $[10-12]$. The development of sports biomechanics is an emerging discipline with broad prospects for development. In the current academic circles, the interest in sports biomechanics is very high, showing a positive state of affairs [13]. According to the results of our previous literature research, through the synthesis of a large number of domestic and 
foreign literatures related to the research direction of sports biomechanics, from the current research status, the current research on sports biomechanics mainly focuses on basic theoretical research. Actions in actual training sports require multiple simulations and guidance in postures and motion states. Research and discussion on teaching methods, etc., involving a series of sports factors including athletes, movements, and sports equipment $[14,15]$.

At present, the research of sports biomechanics mainly focuses on the theoretical research of basic aspects and lacks the new technology of sports biomechanics digital simulation innovation and data measurement [16]. This article takes the badminton chopping action as the research object and carries out biomechanical simulation and simulation research with the help of edge computing and genetic algorithm. The research will provide a reference and reference for the academic circles to explore the field of sports biomechanics.

\section{Related Overview}

2.1. Edge Computing. As an emerging technology, mobile edge computing was first proposed by ETSI and has now become a research hotspot in academia and industry $[17,18]$. In recent years, research on task scheduling strategies in mobile edge computing has also achieved certain results. Research on task scheduling can be roughly divided into device task offloading strategy, edge cloud task scheduling strategy, and task coordination scheduling strategy between the device and the edge cloud. In the MEC network architecture, computing and storage capabilities are delegated to the edge of the mobile network, such as base stations and wireless access points $[19,20]$. Mobile devices can offload application tasks to nearby computing nodes for processing, thereby obtaining low-latency and high-reliability computing services. In the $5 \mathrm{G}$ mobile network, the basic network architecture of MEC consists of three parts: mobile devices, edge cloud, and core network [21-23].

In addition to the breakthrough of basic theory and the reform of digital simulation technology, the innovation in the field of sports biomechanics in my country has also made breakthroughs in the practical application of sports biomechanics. Biomechanics diagnosis technology is the innovation of its application model. Based on big data platform and highspeed networked information means, it realizes the collection of information on the competition venue and training venue and the evaluation of the overall sports state of athletes $[24,25]$. Figure 1 shows the innovation path of biomechanical diagnosis technology based on edge computing.

At present, sports biomechanics diagnosis technology is integrated with multiple disciplines, such as the development of wearable devices, visual analysis, and smart monitoring based on big data, which can achieve precise and personalized diagnosis for different competition types and different sports people [26].

\subsection{Overview of the Development of Sports Mechanics.} The development of sports biomechanics has experienced the initial generalization of simple motor functions with anatomy and mechanics as the core $[27,28]$. Later, in the 18 th century, there has been an exploration of the essence and principle of specific forms of motion, until the exploration of forms of motion with human sports as the core. In fact, the study of human body motion biomechanics relies on the rise of sports, the expansion of fast-developing computer technology, and the breakthrough of simulation experiment technology. The research covers athletes' technical movement simulation research, technical analysis research, conceptual connotation research, mechanics principal research, digital simulation technology research, theoretical practice research, training research, teaching research, etc. $[29,30]$. The biomechanical research content of badminton chopping and hanging action is shown in Figure 2 .

The development of modern sports mechanics does not use the nervous system, muscle system, and bone and joint system as the main research methods [31, 32]. The research also actively introduced quantifiable mathematical elements, using space, numbers, and motion trajectories as elements to construct a mathematical model, using coordinates as the evaluation of the human body's front, back, left, and right torso directions, and using numbers as an explanation of the movement process $[33,34]$. The dynamic equation model is used for comprehensive analysis and query. This method is essentially a simulation and analysis method of the actual human motion, and it is the main direction of current development. Through a large number of literature searches in the early stage, from the current research situation, the current development of sports mechanics is still concentrated on the simulation and comprehensive analysis of athletes' technical movements and the in-depth research on the basic concepts and basic theories of sports biomechanics [35]. The research involves sports equipment research, sports biomechanics training methods, and sports biomechanics teaching methods. Figure 3 shows the framework of the biomechanical innovation model of badminton splitting and hanging.

In the field of physiology, the current innovative research includes differentiated and refined analysis of the individual body physique [36]. It mainly proposes that, in addition to real-time monitoring of basic physiological indicators such as muscle tension, heartbeat, blood pressure, blood lipids, and blood sugar, it is specifically proposed for the monitoring of blood lactic acid, to comprehensively evaluate the athletes' anaerobic glycolysis and acid tolerance, to evaluate the impact of exercise intensity and exercise duration on human blood lactic acid levels, and to pay attention to blood lactic acid levels during normal training and competition. The specific differences: in the field of mechanics, the most innovative research currently is the study of muscle mechanics. After breaking through the limitations of Newtonian mechanics in vitro sports mechanics, muscle mechanics is mainly based on muscles as the main research object, with the Hill equation as the main calculation formula, muscle tension and muscle strength as measurement indicators to explore the in-body muscles during exercise the model of mechanics, explore its synergy and recovery effect, etc., and to try new fields of mechanics. We have introduced 


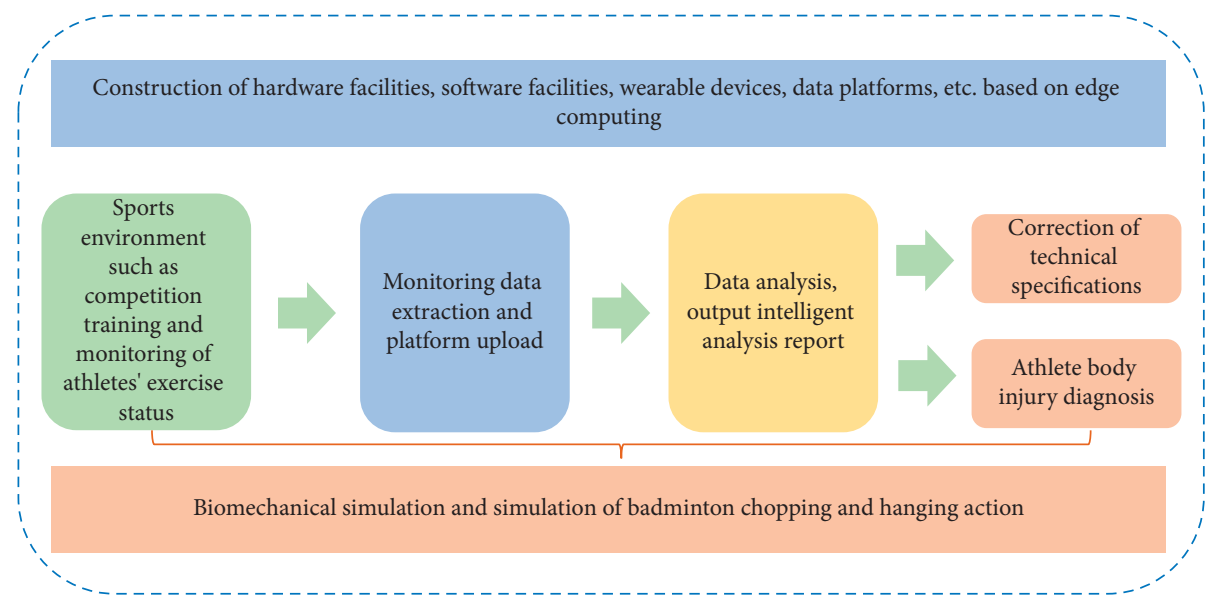

FIGURE 1: Innovation path of biomechanical diagnosis technology based on edge computing.

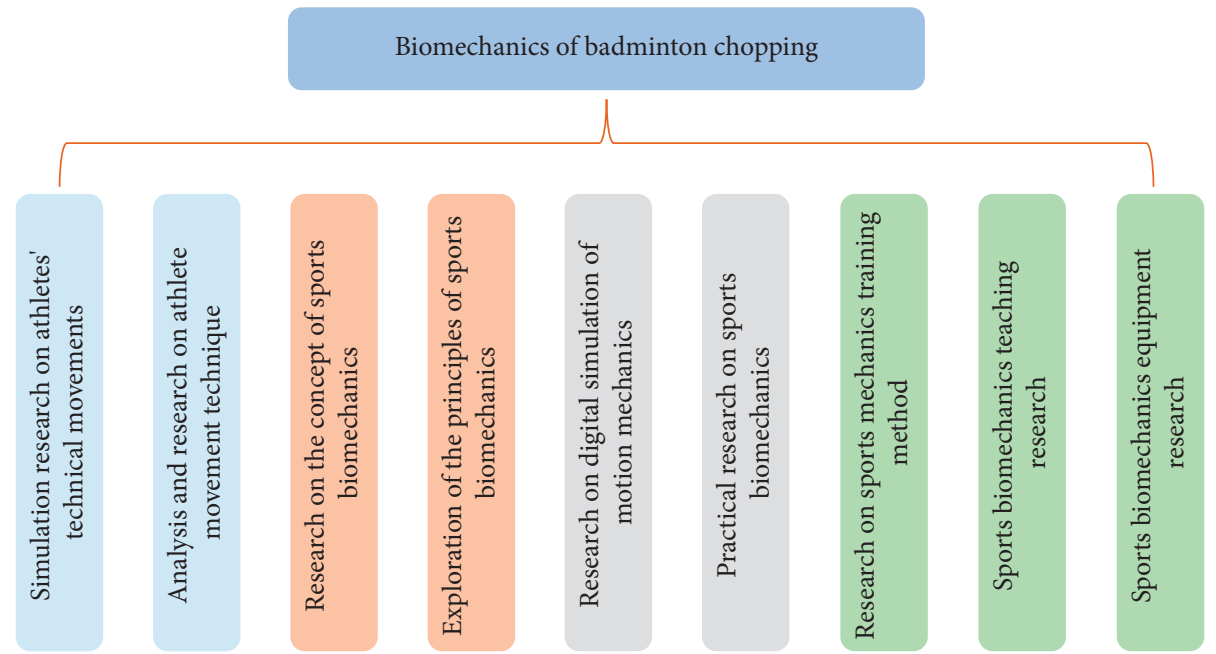

FIgURE 2: Biomechanical research content of badminton chopping and hanging action.

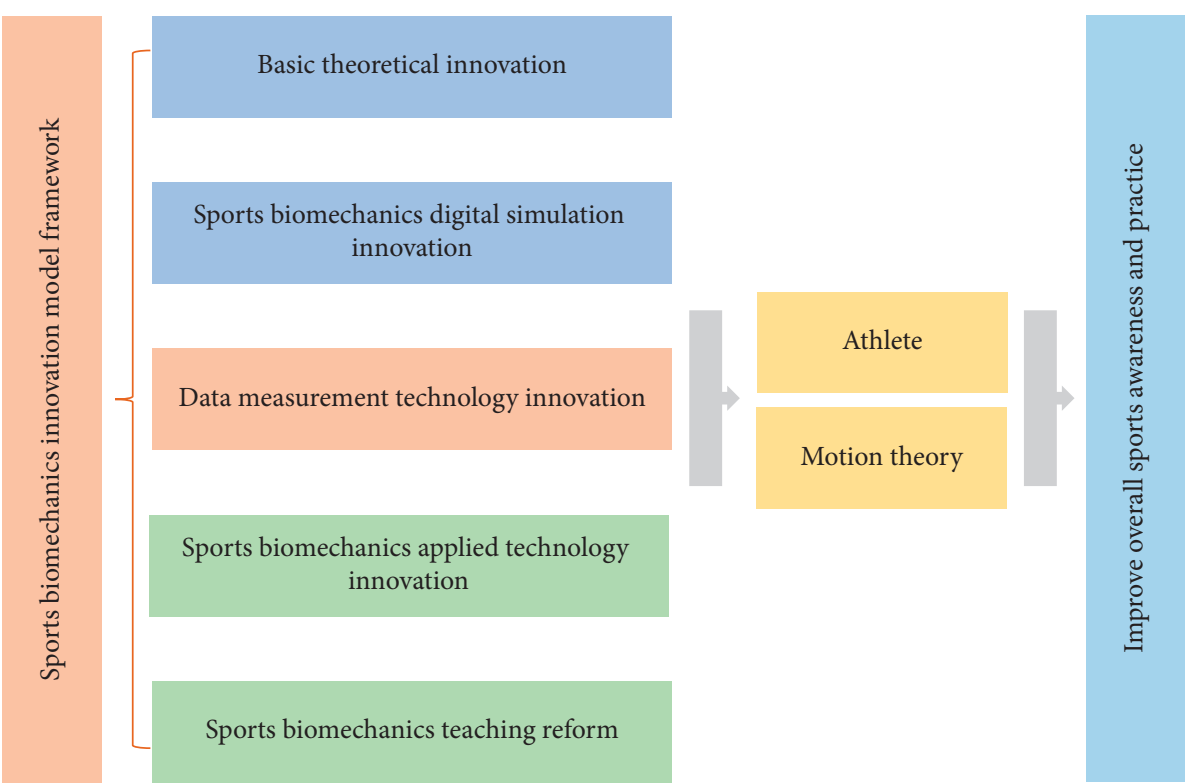

FIGURE 3: Framework of the biomechanical innovation model of badminton chopping and hanging. 
the content of sports biomechanics and the current status of development.

\section{Biomechanical Simulation of Badminton Chopping and Hanging Action Based on Genetic Algorithm}

Sports biomechanics digital simulation innovation is mainly based on new mathematical functions, mathematical formulas, new computer algorithms, and matrix equations as the main research objects. The fusion smoothing algorithm of the unscented Kalman filter is currently a representative digital simulation innovation. In the traditional algorithm, two-dimensional coordinates are mainly used for tracking and analysis. However, the tracking and practice of motion needs to be simulated and analyzed by a three-dimensional algorithm. There must be nonlinear changes and digital modeling under the guidance of nonlinearities. The variables that exist in the measurement of the human body's motion state include state variables and random variables. The distribution of variables will take into account the Gaussian distribution and the nonlinear distribution and cover the phenomenon of matrix transition and mean shift. The introduction of innovative algorithms can accurately and steadily track the human body's posture in the process of complex calculations, reflect the motion characteristics in real time, and achieve accurate assessment of the human motion state.

The basic idea of the genetic algorithm is to start with a set of feasible solutions to the optimization problem, according to the principle of survival of the fittest and survival of the fittest, and evolve from generation to generation to produce better and better feasible solutions. In each generation, according to the fitness of the feasible solution, a part of the good body is selected to be copied to the next generation, and operations such as crossover are performed to generate a set representing the new solution. The optimal individual in the whole process is the optimal solution. Design a new task pricing scheme according to the requirements of the topic, that is, a problem of eliminating the factors of small relevance. Therefore, when designing the fitness function [5], the original objective function value is used as the fitness value to ensure that excellent individuals have a large adaptation value. The target value can be converted into a fitness value through the scale transformation of the following formula, namely,

$$
\text { Fitness }(I)=\frac{D_{\max }-D_{i}+\alpha}{D_{\max }-D_{\min }+\alpha},
$$

where $I$ is the first chromosome of the current population, Fitness $(I)$ is the fitness function value, $D_{\max }$ is the minimum target value of the current population, is the minimum target value of the current population, $D_{\min }$ is the target value to be converted, and $D_{i}$ is the open interval $(0,1)$ positive real numbers within.

The roulette operation is used to select the fitness value. First, generate a random number $\alpha(0 \leq \alpha \leq 1)$ and then choose according to the following formula:

$$
\sum_{j=1}^{n} \frac{f\left(x^{j-1}\right)}{\sum_{n=1}^{\text {pop-size }} f\left(x^{j-1}\right)} \leq \alpha \leq \sum_{j=1}^{n} \frac{f\left(x^{j}\right)}{\sum_{n=1}^{\text {pop-size }} f\left(x^{j}\right)} .
$$

Through this operation, the parent population that needs to be reproduced can be selected. Using single-point crossover and uniform mutation operators, the calculation expressions for crossover and mutation probability are as follows:

$$
\begin{gathered}
p_{c} \begin{cases}k_{2}, & f^{\prime} \leq f_{\mathrm{avg}}, \\
\frac{k_{1}\left(f_{\max }-f^{\prime}\right)}{f_{\max }-f_{\mathrm{avg}}}, & f^{\prime} \geq f_{\mathrm{avg}},\end{cases} \\
p_{m} \begin{cases}k_{4}, & f \leq f_{\mathrm{avg}}, \\
\frac{k_{3}\left(f_{\max }-f\right)}{f_{\max }-f_{\mathrm{avg}}}, & f \geq f_{\mathrm{avg}},\end{cases}
\end{gathered}
$$

where $f_{\max }$ is the largest fitness value in the group, $f_{\text {avg }}$ is the average fitness value of each generation of the group, $f^{\prime}$ is the larger fitness value of the two individuals to be crossed, and $f$ is the fitness value of the individual to be mutated.

$$
X_{i j}=\sum_{n=1}^{5} X_{i j e}
$$

The evaluation index of all evaluation experts is $V_{i j}$, and the gray evaluation right of the first evaluation gray category is $r_{i j e}$ :

$$
r_{i j e}=\frac{X_{i j e}}{X_{i j}} .
$$

Evaluation index $V_{i j}$ is the gray evaluation weight vector $r i j=(r i j 1, r i j 2, r i j 3)$ for each gray category. Synthesize the gray evaluation weight vector, and obtain the gray evaluation weight matrix $R_{i}$ :

$$
R_{i}=\left\{\begin{array}{c}
r i 1 \\
r i 2 \\
\cdots \\
r i j
\end{array}\right\}=\left\{\begin{array}{ccc}
r i 11 & r i 12 & r i 13 \\
r i 21 & r i 22 & r i 23 \\
\ldots & \ldots & \ldots \\
r i j 1 & r i j 2 & r i j 3
\end{array}\right\} .
$$

If the difference in fitness values between chromosomes is large, the fitness value ratio selection is adopted; if the interval is relatively small, the selection tends to be randomly selected among competing chromosomes. Next, focus on the current innovative research in sports biomechanics, try to build an innovative model framework covering new ideas and methods, and achieve breakthroughs in the field of sports biomechanics from multiple dimensions and multiple system levels. Our overall framework mainly covers basic theoretical innovation, sports biomechanics digital simulation innovation, sports biomechanics data measurement and measurement technology, sports biomechanics application technology innovation, sports biomechanics teaching reform, etc. and strives to achieve the depth of sports biomechanics innovation the study. 


\section{Badminton Biomechanics Test Analysis}

In the study of sports biomechanics, data capture and measurement technology is the most critical. Only by realizing the data-based capture and measurement in the early stage can the subsequent digital modeling, matrix analysis, algorithm evaluation, and other series of sports mechanics research be realized. The innovation of sports biomechanics measurement data currently includes balance force testing technology, muscle tension testing technology, stress testing technology, electrophysiological testing technology, medical testing technology, infrared testing technology, and ultrahigh-speed motion capture technology. Figure 4 shows the biomechanical test results of badminton splitting and hanging.

At present, the research field of athlete's technical action simulation in our country is mainly based on camera photography capture technology as the main method. Explore the capture of athletes' movements by different equipment and equipment, as well as possible problems and improvement methods in the process. Figure 5 shows the impact performance test of the badminton split-hanging system.

Sports biomechanics digital simulation technology: the connotation of sports biomechanics is for the study of physiology and mechanics during exercise. Its main research method is to introduce mathematical models or mathematical formulas for deduction. Therefore, digital simulation technology is sports biology. The focus of mechanic's research: at present, the main research focuses on the exploration of classical algorithms, matrix models, and measurement methods in mathematics and the introduction of artificial intelligence, high-speed computers, and simulation technology to enrich the research in this field. Figure 6 shows the simulation test result of the spatial distribution of badminton splitting and hanging motion.

Research on basic concepts and basic theory of sports biomechanics: in the research of kinematics and zoology, the research involving basic concepts and basic theoretical knowledge is also extremely rich. The basic research of sports biomechanics is basically based on the body's physical motor functions including the nervous system, muscle tissue, and skeletal joint system, as well as the categories of gravity, friction, force, and reaction in the field of physics. It is basically a further expansion of the field of mechanics and physiology. Figure 7 shows the simulation results of muscle tension performance of badminton splitting and hanging.

Research on sports biomechanics training methods: theoretical research and breakthroughs are to better guide practice and application, so training methods based on competition and fitness as the main purpose are also the focus of current sports biomechanics research. Under the guidance of sports biomechanics theory, professional athletes in sports competitions are trained to improve the scientific, durability, and explosive power of athletes' sports and, at the same time, take into account the physical and psychological state of athletes. The comparison between genetic algorithm and Bi-LSTM, gradient descent decision tree, and other methods is shown in Figure 8.

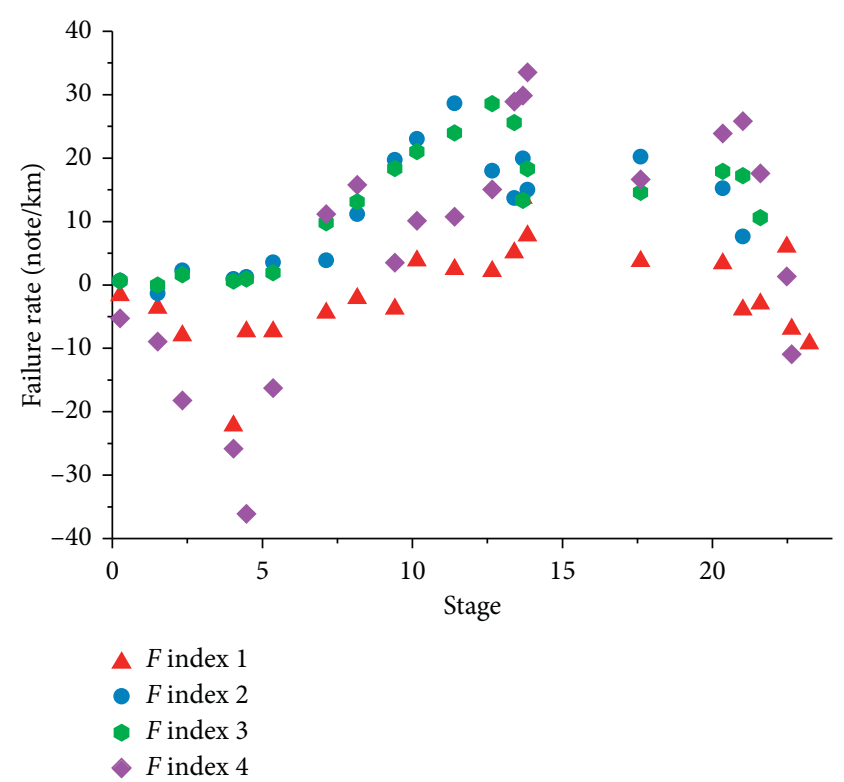

FIGURE 4: Badminton splitting and hanging balance mechanics test results.

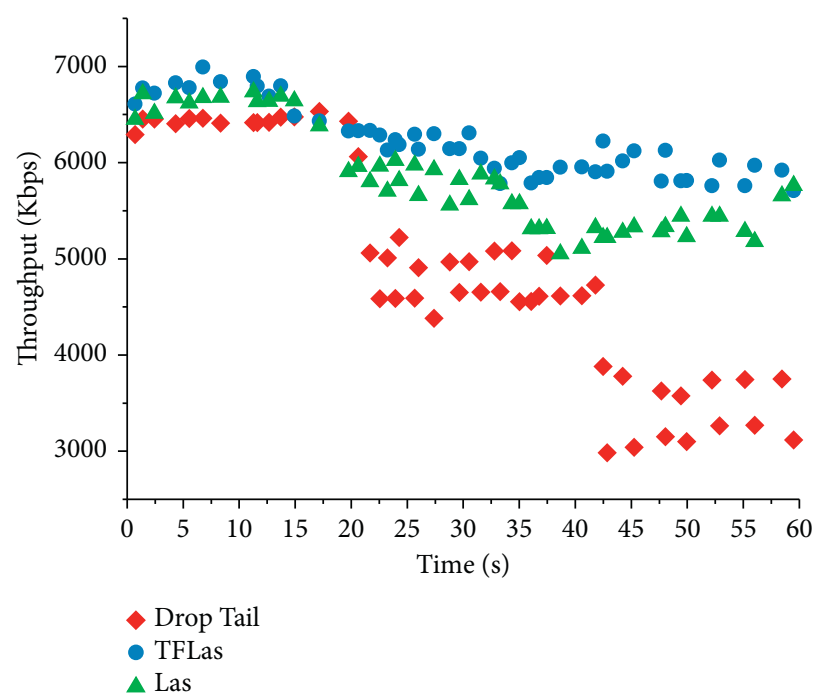

FIGURE 5: Impact performance test of badminton split-hanging system.

In this paper, genetic algorithm is applied to biomechanical simulation, simulation optimization, and data analysis optimization process. In addition, the method is compared with Bi-LSTM, gradient descent decision tree, and other methods. We use the data optimization accuracy rate as an indicator, and the comparison result is shown in Figure 7. Genetic algorithm is better than Bi-LSTM and gradient descent decision tree in the direction of sports biomechanics data optimization [37, 38].System testing and simulation verify the superiority of the method established in this paper. The biomechanical simulation of badminton cutting and suspension motion established by this method has excellent performance. 


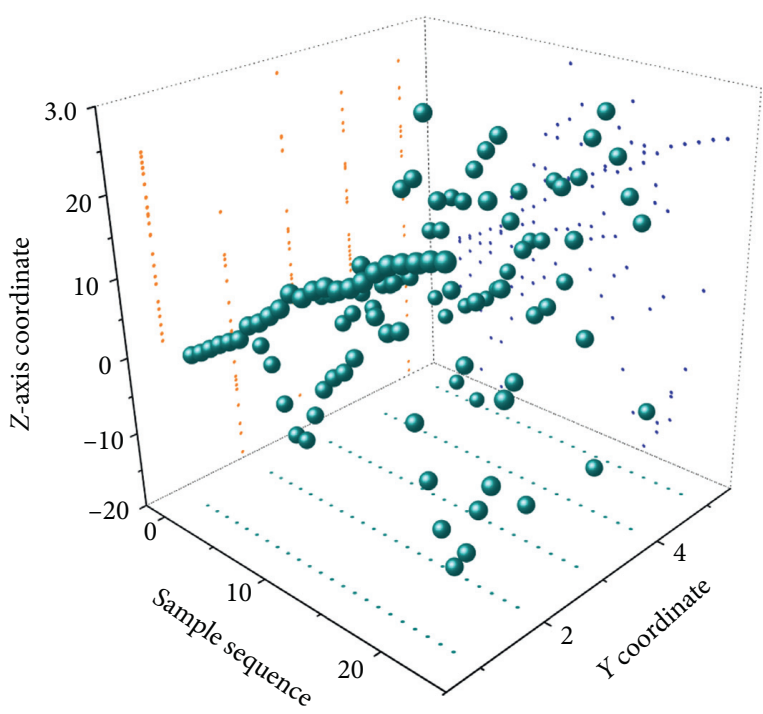

FIGURE 6: Simulation test of the spatial distribution of badminton splitting and hanging movement.

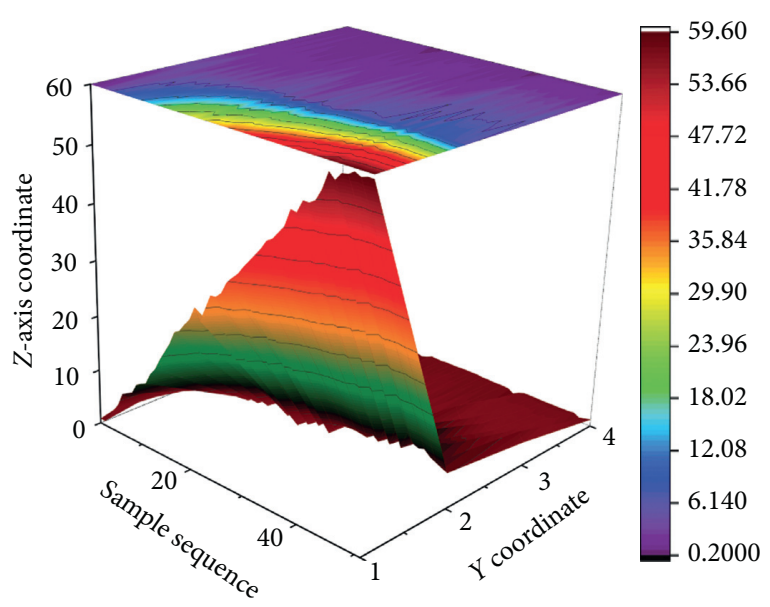

FIGURE 7: Simulation results of muscle tension performance of badminton splitting.

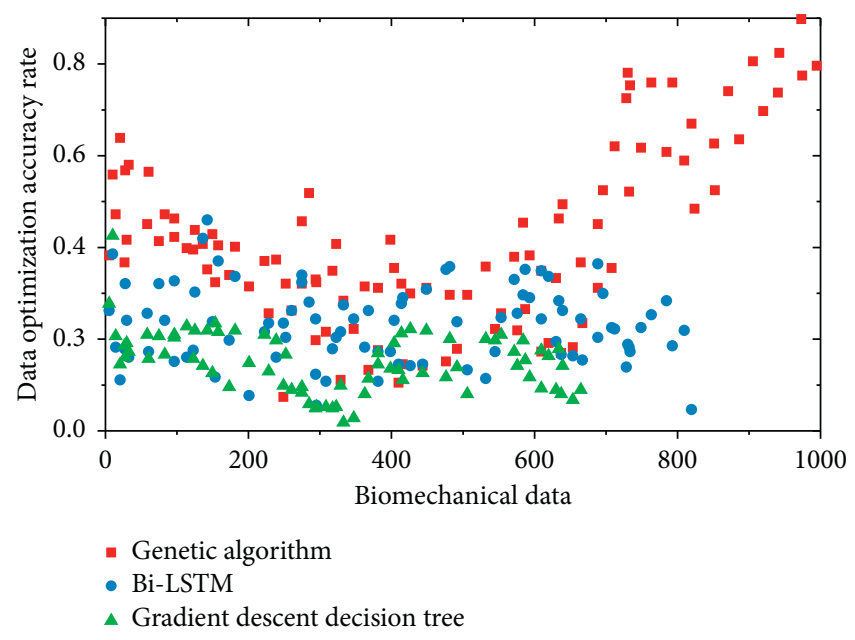

FIGURE 8: Comparison of genetic algorithm with Bi-LSTM, gradient descent decision tree, and other methods.

\section{Conclusion}

At present, a large number of new methods and methods have emerged in the teaching reform of sports biomechanics, mainly focusing on breakthroughs in teaching methods. At present, the importance of sports biomechanics in sports education is increasing day by day, but there are problems such as single teaching methods, solid thinking, and unmatched hardware and software equipment. In short, the innovation of teaching methods allows athletes to have a deep understanding of the biomechanical principles of their own sports and to better realize the evaluation of sports status and the cultivation of athletic ability. In the current research field of sports biomechanics, thanks to the vigorous development of high-precision technology and continuous breakthroughs in biophysiological theory, multidisciplinary can be integrated with sports biomechanics for innovation and development, such as virtual simulation technology and artificial intelligence. It can be combined with sports biomechanics to continuously open up new research fields and realize the development and application of new technologies and methods. We propose a good development prospect for the application of this field in the actual sports process, the changes in the physiological function of the athlete's overall sports state, and the expansion of various sports fields and sports trends, which are conducive to continuous breakthroughs in the field of sports biomechanics.

\section{Data Availability}

The datasets used and/or analyzed during the current study are available from the corresponding author on reasonable request.

\section{Conflicts of Interest}

The author declares that he has no conflicts of interest.

\section{References}

[1] C. Michie, I. Andonovic, C. Davison, A. Hamilton, and M. Gilroy, "The Internet of Things enhancing animal welfare and farm operational efficiency," Journal of Dairy Research, vol. 87 , no. 1 , pp. 1-8, 2020.

[2] N. Matheusara, L. Hernández-Ramosjosé, and F. Skarmetaantonio, "Baldinigianmarco, A survey of cybersecurity certification for the internet of things," Acm Computing Surveys, vol. 23, no. 3, pp. 233-245, 2020.

[3] M. Tozakidou, I. Apine, K. U. Petersen et al., "Comparison of different iterative CT reconstruction techniques and filtered back projection for assessment of the medial clavicular epiphysis in forensic age estimation," International Journal of Legal Medicine, vol. 134, no. 1, pp. 355-361, 2020.

[4] S. Luo, L. Zheng, S. Luo, N. Gu, and X. Tang, "Data sustained misalignment correction in microscopic cone beam CT via optimization under the Grangeat Epipolar consistency condition," Medical Physics, vol. 47, no. 2, pp. 45-58, 2020.

[5] J. Liu, H. R. Zhao, H. L. Wei et al., "Efficacy of bronchoalveolar lavage as adjunct therapy in the treatment of neonatal severe pneumonia: a prospective case-control study," Journal of Tropical Pediatrics, vol. 34, no. 5, pp. 532-546, 2020. 
[6] J. Liu, H. R. Zhao, H. L. Wei, C. Chen, and Y. Q. Gao, "Efficacy of bronchoalveolar lavage as adjunct therapy in the treatment of neonatal severe pneumonia: a prospective case-control study," Journal of Tropical Pediatrics, vol. 19, no. 34, pp. 612-625, 2020.

[7] F. Liu, L. Lei, L. Haitao, S. Liping, and P. Longkai, “The level of plasm donor-derived cell-free dna in kidney transplant patients with severe pneumonia," Transplantation, vol. 12, no. 9, pp. 154-167, 2020.

[8] F. Kassem and G. Kang, "Shin, security and privacy in the internet of things," Computer, vol. 43, no. 13, pp. 765-778, 2019.

[9] H. Jiang, Z. Xiao, Z. Li, J. Xu, and D. Wang, "An energyefficient framework for internet of things underlaying heterogeneous small cell networks," IEEE Transactions on Mobile Computing, vol. 22, no. 99, pp. 413-425, 2020.

[10] J. Huang, J. Guo, H. Li, W. Huang, and T. Zhang, "Efficacy and safety of adjunctive corticosteroids therapy for patients with severe community-acquired pneumonia: a systematic review and meta-analysis," Medicine, vol. 98, no. 13, pp. 235-246, 2019.

[11] P. Guilpain, C. L. Bihan, V. Foulongne, P. Taourel, and V. L. Moing, "Rituximab for granulomatosis with polyangiitis in the pandemic of covid-19: lessons from a case with severe pneumonia," Annals of the Rheumatic Diseases, vol. 2020, pp. 537-549, 2020.

[12] M. D. Greg Zaharchuk and S. Guido Davidzon, "AI for optimization and interpretation of PET/CT and PET/MR images-ScienceDirect," Seminars in Nuclear Medicine, vol. 43, no. 11, pp. 127-134, 2020.

[13] L. Fichera, G. Li-Destri, and N. Tuccitto, "Fluorescent nanoparticle-based Internet of things," Nanoscale, vol. 32, no. 14, pp. 212-234, 2020.

[14] H. Eric and J. Paulina, "Internet of things: energy boon or bane?" Science, vol. 43, no. 14, pp. 323-344, 2019.

[15] A. M. Dimmitt, J. A. Pelz, M. E. Albertson et al., "Evaluation of adaptive statistical iterative reconstruction- $\mathrm{V}$ reconstruction algorithm vs filtered back projection in the detection of hypodense liver lesions: reader performance and preferences," Journal of Computer Assisted Tomography, vol. 43, no. 12, pp. 231-245, 2019.

[16] Z. Deng, H. U. Yuxing, P. Yang, P. Zheng, and X. Tian, "Diagnosis and treatment of an acute severe pneumonia patient with COVID: case report," Journal of Medical Virology, vol. 63, no. 14, pp. 412-424, 2020.

[17] Z. Deng, Y. Hu, P. Yang et al., "Diagnosis and treatment of an acute severe pneumonia patient with COVID-19: case report," Journal of Medical Virology, vol. 17, no. 13, pp. 2405-2412, 2020.

[18] K. Cho, J. Chae, R.-Y. Kwon, S.-C. Bong, and K.-S. Cho, "The application of the filtered backprojection algorithm to solar rotational tomography," The Astrophysical Journal, vol. 895, no. 1, pp. 55-67, 2020.

[19] W.-C. Chen, J.-S. Niu, I.-P. Liu et al., "Study of a palladium (Pd)/Aluminum-Doped zinc oxide (AZO) hydrogen sensor and the kalman algorithm for internet-of-things (IoT) application," IEEE Transactions on Electron Devices, vol. 67, no. 10, pp. 4405-4412, 2020.

[20] J. Chauhan and P. Goswami, "An integrated metaheuristic technique-based energy aware clustering protocol for Internet of Things based smart classroom," Modern Physics Letters B, vol. 12, no. 1, pp. 205-220, 2020.

[21] V. Chang, V. M. Muoz, and M. Ramachandran, "Emerging applications of internet of things, big data, security, and complexity: special issue on collaboration opportunity for IoTBDS and COMPLEXIS," Computing, vol. 43, no. 2, pp. 323-341, 2020.

[22] R. Ceipek, J. Hautz, A. D. Massis, K. Matzler, and L. Ardito, "Digital transformation through exploratory and exploitative internet of things innovations: the impact of family management and technological diversification," Journal of Product Innovation Management, vol. 23, no. 7, pp. 143-156, 2020.

[23] D. T. Braddock, P. R. Stabach, K. Zimmerman, D. Kavanagh, C. T. Sauei, and K. J. Yarema, "Protein engineering and glycan optimization improves pharmicokinetics of an enzyme biologic 10-fold," Faseb Journal, vol. 33, no. 12, pp. 125-136, 2019.

[24] R. Boudewijns, Z. Wang, J. V. Weyenbergh, S. Kaptein, and H. J. Thibaut, "STAT2 signaling restricts viral dissemination but drives severe pneumonia in SARS-CoV-2 infected hamsters," Nature Communications, vol. 11, no. 4, pp. 58-74, 2020.

[25] D. Blez, A. Soulier, F. Bonnet, E. Gayat, and M. Garnier, "Monitoring of high-flow nasal cannula for SARS-CoV-2 severe pneumonia: less is more, better look at respiratory rate," Intensive Care Medicine, vol. 13, no. 4, pp. 160-184, 2020.

[26] M. Beckmann, P. Maass, and J. Nickel, "Error analysis for filtered back projection reconstructions in Besov spaces," Inverse Problems, vol. 37, no. 1, 2020.

[27] O. Barker, "Realizing the promise of the internet of things in smart buildings," Computer, vol. 53, no. 2, pp. 76-79, 2020.

[28] M.-P. Hosseini, T. X. Tran, D. Pompili, K. Elisevich, and H. Soltanian-Zadeh, "Multimodal data analysis of epileptic EEG and rs-fMRI via deep learning and edge computing," Artificial Intelligence in Medicine, vol. 104, 2020.

[29] M. A. Guillén, A. Llanes, B. Imbernón, R. Martínez-Espaa, and J. M. Cecilia, "Performance evaluation of edge-computing platforms for the prediction of low temperatures in agriculture using deep learning," Journal of Supercomputing, vol. 77, no. 9, pp. 145-153, 2021.

[30] M. Gairing and R. Savani, "Computing stable outcomes in symmetric additively separable hedonic games," Mathematics of Operations Research, vol. 12, no. 3, pp. 321-330, 2019.

[31] J. J. Fyfe and J. P. Loenneke, "Interpreting adaptation to concurrent compared with single-mode exercise training: some methodological considerations," Sports Medicine, vol. 48, no. 2, pp. 289-297, 2018.

[32] R. Du, Y. Liu, L. Liu, and W. Du, "A lightweight heterogeneous network clustering algorithm based on edge computing for 5G," Wireless Networks, vol. 26, no. 3, pp. 145-153, 2020.

[33] R. Du, Y. Liu, L. Liu, and W. Du, "A lightweight heterogeneous network clustering algorithm based on edge computing for 5G," Wireless Networks, vol. 26, no. 3, pp. 145-153, 2020.

[34] P. Derbeko, S. Dolev, and E. Gudes, "Wavelet-based dynamic and privacy-preserving similitude data models for edge computing," Wireless Networks, vol. 23, pp. 1-16, 2020.

[35] C.-H. Huang, L.-I. Hsu, T.-K. Chang et al., "Stress distribution of the patellofemoral joint in the anatomic V-shape and curved dome-shape femoral component: a comparison of resurfaced and unresurfaced patellae," Knee Surgery, Sports Traumatology, Arthroscopy, vol. 25, no. 1, pp. 263-271, 2017. 
[36] P. E. Boyle, M. D. Richardson, M. C. Savin, D. E. Karcher, and D. A. Potter, "Ecology and management of earthworm casting on sports turf," Pest Management Science, vol. 75, no. 8, pp. 145-153, 2019.

[37] H. Zheng, F. Lin, X. Feng, and Y. Chen, "A hybrid deep learning model with attention-based conv-LSTM networks for short-term traffic flow prediction," IEEE Transactions on Intelligent Transportation Systems, vol. 99, pp. 1-11, 2020.

[38] Y. Wei, M.-M. Zhao, M. Hong, M.-J. Zhao, and M. Lei, "Learned conjugate gradient descent network for massive MIMO detection," IEEE Transactions on Signal Processing, vol. 68, pp. 6336-6349, 2020. 